\title{
Do weekend oocyte retrievals have an impact on the intracytoplasmic sperm injection cycles outcome?
}

\author{
Amanda Souza Setti ${ }^{1}$, Daniela Paes de Almeida Ferreira Braga ${ }^{1,2}$, Rita de Cássia Savio Figueira ${ }^{2}$, \\ Assumpto laconelli Jr' , Edson Borges J $\mathrm{r}^{1,2}$
}

\author{
1Sapientiae Institute - Educational and Research Center in Assisted Reproduction, \\ São Paulo, Brazil \\ 2Fertility - Assisted Fertilization Center, São Paulo, Brazil
}

Submitted: 4 August 2010

Accepted: 24 November 2010

Arch Med Sci 2012; 8, 2: 368-370

DOI: $10.5114 /$ aoms.2012.28569

Copyright $\odot 2012$ Termedia \& Banach

\begin{abstract}
Introduction: Despite the fact that ovarian stimulation is controlled, it is not always predictable. Because the day of human chorionic gonadotrophin (hCG) injection depends mainly on the patient's ovarian response to gonadotrophins, the day of oocyte retrieval cannot be determined in advance. As a result, oocyte retrievals are often scheduled to occur on weekends, a fact that entails at least one extra working day for the staff, and could lead to physical and psychological stress, especially in embryologists. The aim of this study was to evaluate whether intracytoplasmic sperm injection (ICSI) outcomes are influenced by the day of oocyte retrieval.
\end{abstract}

Material and methods: A total of 327 ICSI cycles, whose retrievals were performed on Wednesdays and Sundays, were analysed in this retrospective study. Cycles were subdivided into two groups according to the day of oocyte retrieval: group $\mathrm{W}(n=196)$, cycles in which oocyte retrieval was performed on Wednesday; and group $S(n=131)$, cycles in which oocyte retrieval was performed on Sunday. Groups were compared regarding fertilization, implantation, pregnancy and take-home baby rates.

Results: No significant differences were observed between groups $A$ and $B$ regarding fertilization rate $(68.9 \%$ and $72.5 \% ; p=0.1589)$, implantation rate $(21.8 \%$ and $24.3 \% ; p=0.5714)$, pregnancy rate $(29.9 \%$ and $31.6 \% ; p=0.7129)$ and take-home baby rate (23.6\% and $28.1 \% ; p=0.4351$ ).

Conclusions: A well-trained embryologist's group adhering to staff scheduling allows large programmes to ensure a similar outcome independent of the workload or workday on which embryologists perform the manipulation of gametes.

Key words: in vitro fertilization, intracytoplasmic sperm injection, workload.

\section{Introduction}

Palermo et al. [1] reported pregnancies and births following intracytoplasmic injection (ICSI) of a single sperm into an oocyte. The ICSI has become a well-established method of in vitro fertilization (IVF) and, over the last decade, the treatment of choice for many couples with untreatable infertility.

The ICSI involves the injection of a single spermatozoon into the cytoplasm of a mature oocyte obtained by ovarian puncture following controlled ovarian stimulation (COS). Since Porter et al. [2] first reported the use of

\author{
Corresponding author: \\ Edson Borges Jr MD, PhD \\ Av. Brigadeiro Luis Antonio \\ 4545 \\ 01401-002 São Paulo \\ SP, Brazil \\ Phone: (55 11) 3018-8181 \\ E-mail: edson@fertility.com.br
}


gonadotrophins combined with gonadotrophinreleasing hormone $(\mathrm{GnRH})$ agonist for ovarian stimulation in IVF procedures, this technique has been widely used in order to maximize pregnancy opportunities. Follicle stimulation hormone (FSH) is administered to stimulate the ovaries to produce a higher number of oocytes in an IVF cycle. This medication is self-administered by the patient and is required for a period of 9-14 days [3]. The follicular development is monitored by serial transvaginal ultrasound examinations and oestradiol (E2) levels. Once the monitoring results indicate mature oocytes (appropriate follicle size and oestrogen levels), the maturation process completion is trigged by the administration of human chorionic gonadotrophin (hCG) and the oocyte retrieval is scheduled for 34-36 $\mathrm{h}$ later [4].

Despite the fact that ovarian stimulation is controlled, it is not always predictable. Because the day of hCG injection depends mainly on the patient's ovarian response to gonadotrophins, the day of oocyte retrieval cannot be determined in advance. As a result, sometimes oocyte retrievals are scheduled to occur on weekends, a fact that entails at least one extra working day for the staff, and could lead to physical and psychological stress, especially in embryologists [5].

The success of the main important step of ICSI, the spermatozoon injection per se, occurs inside the IVF laboratory and relies on the embryologist's hands. In fact, one of the most significant challenges in assisted reproduction technology (ART) is achieving technical proficiency in micromanipulation [6]. Once the proficiency is developed, the embryologist becomes ready to perform the IVF procedures by himself, and to participate in the team's scheduling.

Thus, the IVF staff not only has to work during the week, but also its presence is mandatory whenever there is a cycle programmed for the weekend.

One recent study addressed the avoidance of weekend oocyte retrieval, and concluded that it is possible to safely avoid weekend oocyte retrievals by advancing an ideal Saturday oocyte retrieval to Friday, and delaying an ideal Sunday oocyte retrieval to Monday, without adversely impacting on IVF outcomes [7]. Nevertheless, whether working during the weekend may be a cause of stress and tiredness for the embryologists, leading to the impairment of ICSI outcomes, is still to be elucidated.

The aim of this study was to evaluate whether ICSI outcomes are influenced by the day of oocyte retrieval.

\section{Material and methods}

\section{Experimental design}

Using our private assisted fertilization centre's database, a total of 327 ICSI cycles, performed from
January 2004 to December 2008, whose retrievals were performed on Wednesdays and Sundays, were analysed in this retrospective study. Wednesday and Sunday were elected to represent a weekday and a weekend day respectively. Cycles were subdivided into two groups according to the day of oocyte retrieval: group W $(n=196)$, cycles in which oocyte retrieval was performed on Wednesday; and group $S(n=131)$, cycles in which oocyte retrieval was performed on Sunday. Groups were compared regarding fertilization, implantation, pregnancy and take-home baby rates. The study was approved by the local Institutional Review Board.

\section{Assessment of fertilization and embryo transfer}

Fertilization was assessed $18 \mathrm{~h}$ after ICSI. Normal fertilization was confirmed when two clearly distinct pronuclei were present. Embryo transfer was performed on the third day of development. One to three embryos from each couple were transferred.

\section{Statistical analysis}

Results are expressed as means \pm standard deviation (SD) for numeric variables and proportions (\%) for categorical variables. Mean values were compared using Student's $t$ parametric test or the Mann-Whitney non-parametric test, as appropriate. The proportions were compared by the $\chi^{2}$ test. Results were considered to be significant at the $5 \%$ critical level $(p<0.05)$. Data analysis was carried out using GraphPad Prism version 4.0 statistical program.

\section{Results}

The causes of infertility were evenly distributed in both groups. There were no significant differences regarding the cycle's general characteristics. Similar female age ( $34.5 \pm 4.8$ years vs. $34.4 \pm 5.0$ years, $p=0.8079$ ), total dose of recombinant follicle stimulating hormone administered (2306 \pm 699.3 vs. $2258 \pm 813.8, p=0.2808)$, oestradiol levels on the day of hCG administration (1883 \pm 1847 vs. 1985 $\pm 2183, p=0.8565)$, mean number of aspirated follicles (17.6 \pm 13.5 vs. $16.7 \pm 12.9, p=0.5328)$, oocyte yield $(67.5 \%$ vs. $62.8 \%, p=0.1026)$, MII oocyte rate (71.7\% vs. $69.1 \%, p=0.3598)$ and mean number of transferred embryos ( $2.5 \pm 1.3$ vs. $2.3 \pm 1.3, p=0.3705)$ were observed.

\section{Intracytoplasmic sperm injection outcome}

No significant differences were observed between group $\mathrm{W}$ and group $\mathrm{S}$ regarding fertilization rate $(68.9 \%$ vs. $72.5 \%, p=0.1589)$, implantation rate $(21.8 \%$ vs. $24.3, p=0.5714)$, pregnancy rate (29.9\% vs. $31.6 \%, p=0.7129)$ and take-home baby rate $(23.6 \%$ vs. $28.1 \%, p=0.4351)$. 


\section{Discussion}

The day of ICSI performance does not seem to interfere with ICSI outcome. No significant differences were found between the groups of patients that have had their ICSI cycles performed on a weekday and on the weekend regarding all the evaluated variables. In fact, there was a slight trend towards better results in patients who underwent weekend cycles. This finding could possibly be explained by the smaller number of patients programmed to undergo oocyte retrievals on the same weekend day, and due to the fact that staff scheduling could not only overcome their tiredness and stress, but also be reflected in fewer nurses, physicians and embryologists involved in the in vitro fertilization procedures.

One similar retrospective study, comprising a total of 1558 IVF laboratory procedures, attempted to ascertain whether the daily activity in the IVF laboratory is related to fertilization and pregnancy rates. The daily workload was divided into three categories: optimal, overload, and high overload, according to the number of procedures performed within a day. The authors reported no adverse effects associated with the daily workload [8].

Unlike most medical laboratories that play a diagnostic role, laboratories for ART are involved in the treatment of infertile couples. Certainly for ART centres, the laboratory is one of the most critical environments. Handling human gametes and producing human embryos in order to achieve pregnancies constitute the key tasks of an ART laboratory [9]. Indeed, none of the procedures utilized in human IVF are unique or technically very difficult to perform. However, when considering the nature of the material being handled, it is essential that all laboratory procedures are optimal for IVF. The individuals handling the gametes and embryos must be suitably trained and experienced in order to achieve the main goal of the IVF laboratory: to guarantee a constant level of success for every step of all procedures by every staff member involved [10].

Recruitment, training, and motivation of highly qualified people are some of the most important tasks facing the management of an IVF unit. Each individual position must have its tasks and responsibilities clearly stated. It is therefore essential to have a system to ensure that everybody knows exactly how everything should be done. Moreover, the number of staff has to be adjusted according to the number of cycles performed and the nature of procedures performed in the laboratory. Furthermore, the minimum number of employees necessary for the department should be determined. Regulation of holiday breaks and compensation strategies for every position in the laboratory has to be laid down in detail.
Additionally, introducing and fully implementing a quality control system in the laboratory is a perfect manner to standardize the methods and the way that the embryologists perform their work. It has benefits also in optimization of the environment in which the patient's gametes and embryos are handled [11-13].

In conclusion, in the present study, we have outlined the role and importance of implementing suitable staff scheduling in order to ensure high quality and continual improvement in centres striving for excellence. A well-trained embryologist's group adhering to staff scheduling allows large programmes to ensure a similar outcome independent of the workload and workday on which embryologists perform the manipulation of gametes.

\section{References}

1. Palermo G, Joris H, Devroey P, et al. Pregnancies after intracytoplasmic injection of single spermatozoon into an oocyte. Lancet 1992; 340: 17-8.

2. Porter RN, Smith W, Craft IL, et al. Induction of ovulation for in-vitro fertilisation using buserelin and gonadotropins. Lancet 1984; 2: 1284-5.

3. Jennings JC, Moreland K, Peterson CM. In vitro fertilisation. A review of drug therapy and clinical management. Drugs 1996; 52: 313-43.

4. Hillier SG, Afnan AM, Margara RA, et al. Superovulation strategy before in vitro fertilization. Clin Obstet Gynaecol 1985; 12: 687-723.

5. Nakagawa K, Yamano S, Senuma M, et al. Avoidance of oocyte retrieval on the weekend through the use of scheduled ovarian hyperstimulation for in vitro fertilization and embryo transfer. Fertil Steril 1997; 68: 787-90.

6. Braga DP, Pasqualotto FF, Madaschi C, et al. Use of pig oocytes for training new professionals in human assisted reproduction laboratories. Fertil Steril 2007; 88: 1408-12.

7. Tremellen KP, Lane M. Avoidance of weekend oocyte retrievals during $\mathrm{GnRH}$ antagonist treatment by simple advancement or delay of hCG administration does not adversely affect IVF live birth outcomes. Hum Reprod 2010; 25: 1219-1224.

8. Exposito A, Matorras R, Mendoza R, et al. Daily workload in the embryology laboratory and in vitro fertilization results. J Reprod Med 2010; 55: 49-54.

9. Kastrop PM. Quality management in the ART laboratory. Reprod Biomed Online 2003; 7: 691-4.

10. Keck C, Fischer R, Baukloh V, et al. Staff management in the in vitro fertilization laboratory. Fertil Steril 2005; 84: 1786-8.

11. Matson PL. Internal quality control and external quality assurance in the IVF laboratory. Hum Reprod 1998; 13 Suppl 4: 156-65.

12. Wikland $M$, Sjoblom C. The application of quality systems in ART programs. Mol Cell Endocrinol 2000; 166: 3-7.

13. Dandekar PV, Quigley MM. Laboratory setup for human in vitro fertilization. Fertil Steril 1984; 42: 1-12. 